\title{
Gastroprotektion
}

\section{Risiko für NSAR-Läsionen reduzieren}

- Traditionelle nicht steroidale Antirheumatika (tNSAR) sind ebenso wie Cyclooxygenase-(COX-)2-Hemmer aufgrund ihrer analgetischen und antiphlogistischen Wirkung eine "exzellente" Therapieoption bei Arthrose und rheumatoider Arthritis, betonte Prof. Andreas de Werth, Hamburg, mit Verweis auf die MEDAL-Studie. Der Unterschied zwischen den beiden Substanzklassen zeigt sich beim Blick auf die gastrointestinalen Nebenwirkungen: Das Risiko für Perforationen, Ulzera und Blutungen (PUB) ist unter tNSARs deutlich erhöht. So treten gastroduodenale Läsionen unter Naproxen und Ibuprofen häufiger auf als unter Etoricoxib (kumulative Inzidenzrate über zwölf Wochen: 25,7 vs. $7,4 \% ; 17,0$ vs. $8,1 \%$ ). Beim Vergleich von Etoricoxib (Arco$\mathrm{xia}^{\circledR}$ ) mit verschiedenen tNSAR (Naproxen $1000 \mathrm{mg} / \mathrm{d}$, Ibuprofen $2400 \mathrm{mg} / \mathrm{d}$ oder
Diclofenac 150 mg/d) lag die Inzidenz von PUB unter Etoricoxib um 55\% niedriger.

tNSAR kombiniert mit einem Protonenpumpeninhibitor (PPI) reduzieren das Blutungsrisiko ähnlich effektiv wie ein Coxib. "Die Kombination ist nicht unterlegen, aber auch nicht überlegen", so de Werth mit Blick auf eine Studie, die die Häufigkeit rezidivierender Ulkusblutungen unter einem Coxib und Diclofenac plus Omeprazol über sechs Monate verglich. Er betonte, dass NSAR-induzierte Läsionen im Dünndarm durch die Kombi tNSAR plus PPI nicht reduziert werden können. Unter COX-2-Hemmern treten sie dagegen seltener auf.

Eine Primärprophylaxe durch ein Coxib oder eine kombinierte tNSAR/PPI-Therapie ist laut de Werth bei gesicherten Risikofaktoren indiziert: Alter über 60 Jahre, Ulkus in der Anamnese und Steroidtherapie, v.a.

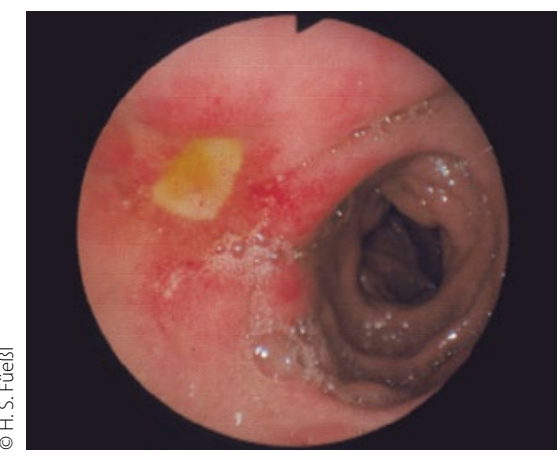

Ulkus im Bulbus duodeni.

auch die Einnahme von Antikoagulanzien, "denn, wenn es bei diesen Patienten blutet, dann richtig". Die zweifelsfrei beste Prophylaxe gastrointestinaler Blutungen ist die Kombination eines Coxibs mit einem PPI. „Damit lässt sich eine Reduktion auf null erreichen."

\footnotetext{
- Dr. Beate Fessler

Quelle: MSD-Symposium „Gelenkschmerzen aus dem Blickwinkel verschiedener Fachrichtungen",
} Wiesbaden, April 2012 (Veranstalter: MSD)
Somatostatin-Analogon gegen Morbus Cushing - Als erste medikamentöse Therapie für Patienten mit Morbus Cushing wurde im April 2012 das Somatostatin-Analogon Pasireotid (Signifor ${ }^{\circledR}$ ) zugelassen. Die Zulassung umfasst die Behandlung von Cushing-Patienten, für die die Standardtherapie, die Resektion des ACTH-sezernierenden Hypophysenadenoms, keine Option ist oder bei denen diese Operation fehlgeschlagen ist. In einer multizentrischen, randomisierten, doppelblinden Phase-II-Studie mit 162 Patienten, die in der Mehrzahl an persistierendem oder rezidiviertem $M$. Cushing litten, gelang nicht nur eine Reduktion der Cortisol-Ausscheidung im 24-Stunden-Urin nach sechs Monaten Therapie mit Pasireotid. Die Therapie führte auch zu einer deutlichen Gewichtsreduktion und Blutdrucksenkung sowie zu einer verbesserten Lebensqualität der Patienten.

Bei $40 \%$ der Patienten wurde unter der Therapie eine Hyperglykämie fest- gestellt, die jedoch laut Prof. Jochen Schopohl, München, therapeutisch gut beherrschbar ist.

- Lauch-Presseveranstaltung, Nürnberg, Mai 2012 (Veranstalter: Novartis)

\section{Monoklonaler Antikörper gegen SLE}

- Erwachsene Patienten mit trotz Standardtherapie aktivem Systemischem Lupus erythematodes (SLE) können jetzt mit dem monoklonalen Antikörper Belimumab (Benlysta ${ }^{\circledR}$ ) behandelt werden. In den BLISS-Studien wurden 865 bzw. 819 Patienten mit aktivem SLE über 52 bzw. 75 Wochen zusätzlich zur Standardtherapie mit entweder $1 \mathrm{mg} / \mathrm{kg}$ oder $10 \mathrm{mg} / \mathrm{kg}$ Belimumab oder Placebo behandelt. In BLISS-52 wurden Ansprechraten von 57,6\% für die 10 mg/kg-Dosis, in BLISS-76 43,2\% in Woche 52 erzielt. Die Verträglichkeit war insgesamt gut.

Der Gemeinsame Bundesausschuss (GBA) hat Belimumab in seiner Sitzung am 2. August 2012 einen Hinweis auf einen beträchtlichen Zusatznutzen attestiert.

- Symposium in Wiesbaden, April 2012 (Veranstalter: GlaxoSmithKline)

MOR-NRI-Analgesie - Das Analgetikum Tapentadol, das zwei Wirkmechanismen vereint, hat sich bei Indikationen wie Arthrose, Rückenschmerzen oder Neuropathien bewährt. Dies hat sich mittlerweile auch im Praxisalltag bestätigt. Im nozizeptiven Schmerzmodell sei der $\mu$-Opioid-RezeptorAgonismus (MOR), im neuropathischen Schmerzmodell die Hemmung der Noradrenalin-Wiederaufnahme (NRI) die entscheidenden Mechanismen. Tapentadol (Palexia ${ }^{\circledR}$ retard) wirkt bei Patienten mit Arthroseschmerz oder Rückenschmerzen ebenso gut wie Oxycodon $\mathrm{CR}$, verursacht aber weniger opioidtypische Nebenwirkungen.

- Pressekonferenz „Tapentadol: zwei Wege ein Ziel“, Frankfurt, März 2012 (Veranstalter: Grünenthal) 